\title{
Le système scolaire français et ses miroirs
}

Les missions pédagogiques entre comparaison internationale et circulation des savoirs (1842-1914)

The French school system and its mirrors. Between international comparisons and the circulation of knowledge - the pedagogical missions (1842-1914)

Das französische Schulsystem und seine Spiegelbilder. Pädagogische Missionen zwischen internationalem Vergleich und Wissensverbreitung (1842-1914) El sistema escolar francés y sus espejos. Las misiones pedagógicas entre comparación internacional y circulación de los saberes (1842-1914)

\section{Damiano Matasci}

\section{OpenEdition}

\section{Journals}

Édition électronique

URL : https://journals.openedition.org/histoire-education/2131

DOI : $10.4000 /$ histoire-education.2131

ISSN : 2102-5452

Éditeur

ENS Éditions

\section{Édition imprimée}

Date de publication : 1 janvier 2010

Pagination : 5-26

ISSN : 0221-6280

\section{Référence électronique}

Damiano Matasci, «Le système scolaire français et ses miroirs », Histoire de l'éducation [En ligne], 125 | 2010, mis en ligne le 01 janvier 2014, consulté le 20 mai 2021. URL : http://journals.openedition.org/ histoire-education/2131; DOI : https://doi.org/10.4000/histoire-education.2131 
Le système scolaire français et ses miroirs

Les missions pédagogiques entre comparaison internationale et circulation des savoirs (1842-1914)

\section{Damiano MATASCI}

Si l'histoire transnationale des pratiques et des savoirs universitaires est relativement bien étudiée, le bilan historiographique des travaux sur les niveaux inférieurs des systèmes éducatifs se présente différemment. Ayant échappé à l'engouement récent pour ce champ d'étude, l'histoire de l'éducation reste encore en grande partie inscrite dans une perspective exclusivement nationale, et pas seulement en France. Or, la mise en place des systèmes scolaires modernes au $\mathrm{XX}^{\mathrm{e}}$ siècle se caractérise par des projets de réformes qui soulèvent une série de questionnements et de défis communs à l'échelle européenne $^{1}$. Les débats sur l'organisation, la fonction sociale et la coordination des différents degrés d'enseignement se trouvent ainsi au centre d'une circulation d'informations traversant les frontières ${ }^{2}$. Par conséquent, la mobilisation

1 Fritz Ringer, Detlef Müller, Brian Simon (dir.), The Rise of the Modern Educational System: Structural Change and Social Reproduction (1870-1920), Cambridge, Cambridge University Press, 1987.

2 Eckhardt Fuchs (dir.), Bildung International. Historische Perspektiven und Aktuelle Entwicklungen, Würzburg, Ergon Verlag, 2006. 
des exemples étrangers constitue une pratique courante lors des processus de réforme, et elle joue de façon permanente dans le cas français ${ }^{3}$.

Tout au long du XIX ${ }^{e}$ siècle, le ministère français de l'Instruction publique charge une multitude d'acteurs, pour la plupart des enseignants et des inspecteurs scolaires, d'une série de voyages afin de recueillir une somme d'informations sur les systèmes scolaires étrangers. Les buts sont d'étudier les nouveaux procédés pédagogiques, les différents problèmes relatifs à l'instruction publique, les solutions diverses qu'on y a apportées, et de mener une réflexion sur la possibilité d'emprunts applicables au système scolaire national. Avec les congrès internationaux de l'enseignement et les sections scolaires des expositions universelles, ces missions représentent l'un des vecteurs de la circulation internationale des idées pédagogiques et permettent de réfléchir à la construction de modèles scolaires de référence, qui façonnent l'évolution des politiques nationales ${ }^{4}$. Si, comme le souligne Christophe Charle, tout échange culturel est effectué à travers des " formes sociales spécifiques " ${ }^{5}$, les missions incarnent un terrain d'étude fort stimulant pour examiner la circulation de flux d'informations transnationaux dans le domaine scolaire.

Cet article propose une étude des missions pédagogiques entre 1842, année de création d'un " service des missions " au sein du ministère de l'Instruction publique, et $1914^{6}$. L'ambition est d'en étudier l'organisation, la fonction, les différents types, ainsi que d'identifier les acteurs promouvant la production d'un savoir sur l'étranger dans le domaine scolaire. L'article privilégie les degrés inférieurs du système scolaire (primaire, secondaire, professionnel), qui sont moins étudiés que l'enseignement supérieur ${ }^{7}$. En outre, plus qu'à une

3 Sur le rôle des " exemples étrangers " dans le façonnement des politiques scolaires nationales, voir Bernard Trouillet, "Der Sieg des preussischen Schulmeisters " und seine Folgen für Frankreich, 1870-1914, Cologne, Böhlau Verlag, 1991, et Philippe Gonon, Das Internationale Argument in der Bildungsreform, Bern, Peter Lang, 1998.

4 Berndt Zymek, Das Ausland als Argument in der Pädagogischen Reformdiskussion, Ratingen, Aloys Henn Verlag, 1975.

5 Christophe Charle, Jürgen Schriewer, Peter Wagner (dir.), Transnational Intellectual Networks. Forms of Academic Knowledge and the Search for Cultural Identities, Francfort/New York, Campus Verlag, 2004, p. 13.

6 Cet article analyse une base de données obtenue grâce au dépouillement systématique de plusieurs fonds d'archives et à leur croisement avec les documents imprimés disponibles à la Bibliothèque nationale de France (monographies et rapports de mission). Le lecteur trouvera les références détaillées dans les notes de bas de page ci-dessous.

7 Pour l'enseignement supérieur, voir Jean-Christophe Bourquin, L'État et les voyageurs savants. Légitimités individuelles et volontés politiques. Les missions du ministère de l'Instruction publique (1840-1914), thèse de doctorat de l'université de Paris 1, sous la dir. d'Antoine Prost, Paris, 1993. 
approche en termes de transferts $^{8}$, qui risque de cloisonner l'analyse dans un processus linéaire d'introduction-diffusion-réception d'un objet ou d'une d'idée, l'article souhaite questionner l'économie d'un " régime circulatoire " ${ }^{9}$ dans le domaine scolaire, en se focalisant sur les acteurs qui alimentent les contacts et les comparaisons internationales. Après avoir rappelé les étapes principales de l'internationalisation de la vie pédagogique française au XIX ${ }^{e}$ siècle, le rôle qu'y tiennent les missions et le dispositif administratif mis en place pour les encadrer, on évoquera les caractéristiques sociales des acteurs, en mettant en évidence les trois différentes catégories de missions, qui reflètent la diversité des profils sociaux et professionnels des "chargés de mission ", ainsi que celle des objets d'étude. Enfin, nous étudierons le rôle des missions dans la construction de modèles scolaires étrangers, ainsi que les critères de sélection des pays de destination.

\section{I - Missions d'étude et circulation des idées pédagogiques au $\mathrm{XIX}^{\mathrm{e}}$ siècle}

La mission scientifique, ou plus généralement le voyage d'étude à l'étranger, représente un élément central de l'internationalisation du discours pédagogique au XIX ${ }^{\mathrm{e}}$ siècle. À cet égard, les missions des professeurs, universitaires et pédagogues anglais ou américains attirent depuis des années l'attention des chercheurs ${ }^{10}$. Soucieux de se renseigner sur les méthodes, les pratiques et les organisations scolaires en vigueur en Europe, ces professeurs traversent le vieux continent en collectant une série d'observations et de renseignements susceptibles d'éclairer leurs propres politiques scolaires et de les inscrire dans un contexte international. Ces explorations pédagogiques alimentent une production de savoirs sur l'étranger qui façonnent une vision autre du contexte scolaire national et stimulent une réflexivité possible uniquement à travers la comparaison avec d'autres expériences. Par ailleurs, les questions pédagogi-

8 Michel Espagne, Michael Werner (dir.), Transferts. Les relations interculturelles dans l'espace francoallemand (XVIII -XIX ${ }^{e}$ siècles), Paris, Éd. Recherche sur les Civilisations, 1988.

9 Pierre-Yves Saunier, "Les régimes circulatoires du domaine social 1800-1940 : projets et ingénierie de la convergence et de la différence ", Genèses, n 71, 2008, p. 4-25.

10 David Phillips, "Mehr als Reiseberichte ? Britische Beobachter des deutschen Bildungswesen im 19. Jahrhundert ", in Jürgen Schriewer (dir.), Weltkultur und kulturelle Bedeutungswelten. Zur Globarisierung von Bildungsdiskursen, New York, Campus Verlag, 2007, p. 23-43. Quelques indications sur les voyages scientifiques français en Allemagne dans Hélène Barbey-Say, Le voyage de France en Allemagne de 1871 à 1914, Nancy, Presses Universitaires de Nancy, 1994. 
ques s'inscrivent dans le mouvement plus général d'internationalisation de la vie scientifique au XIX ${ }^{e}$ siècle, qui va de l'intensification de la circulation des étudiants aux échanges de professeurs entre les universités, à la mise en place de catalogues bibliographiques internationaux et à l'essor des congrès internationaux de l'enseignement.

En France, l'année 1842 marque la création officielle du " service des missions " au sein de la division des sciences et lettres du ministère de l'Instruction publique. Bien que de nombreuses missions aient déjà eu lieu dans les premières décennies du $\mathrm{XIX}^{\mathrm{e}}$ siècle $^{11}$, cette date marque le renforcement du soutien du ministère aux chercheurs désireux d'aller collecter des données scientifiques, géographiques ou archéologiques à l'étranger, dans les bibliothèques, les universités ou les dépôts d'archives. Les activités vont de la recherche de documents historiques aux fouilles archéologiques, des explorations géographiques à l'établissement de catalogues des musées. Plus de 1200 missions littéraires et scientifiques, touchant un nombre substantiel de sujets scientifiques, parcourent alors le continent européen entre 1842 et $1914^{12}$. Dans une perspective plus limitée, c'est-à-dire en circonscrivant l'analyse à l'enseignement primaire, secondaire et professionnel, les recherches entreprises dans plusieurs fonds d'archives ont permis de rassembler les documents relatifs à 131 missions. Concernant 114 personnes, elles touchent un total de 27 pays différents. Le nombre de missions et de pays soumis à une enquête connaît une augmentation remarquable dans la deuxième moitié du XIX ${ }^{e}$ siècle (surtout à partir des années 1870-1880), accompagnée par une plus vaste diversité des destinations (tableau 1).

11 Nous mentionnons ici quelques-unes des plus connues : la tournée des émissaires de l'Empire, Cuvier et Noël, dans les départements tout récemment annexés de la Hollande et de la Basse Allemagne pour y inspecter les établissements d'instruction publique (deux rapports publiés en 1811), la mission de Victor Cousin sur l'instruction publique en Prusse en 1831, ainsi que celle de SaintMarc Girardin dans le midi de l'Allemagne et en Suisse (1835-1839).

12 Jean-Christophe Bourquin, "National Influences on International Scientific Activity: The Case of the French Missions Littéraires in Europe, 1842-1914 ", in Christophe Charle, Jürgen Schriewer, Peter Wagner (dir.), op. cit., p. 451. 
Tableau 1. Distribution chronologique des missions et diversité des destinations, $1842-1914^{13}$.

\begin{tabular}{|l|c|c|c|}
\hline Années & Nombre de missions & $\begin{array}{l}\text { Nombre de pays } \\
\text { différents visités }\end{array}$ & Nombre total de visites \\
\hline $1842-1850$ & 7 & 7 & 11 \\
\hline $1851-1860$ & 4 & 5 & 7 \\
\hline $1861-1870$ & 15 & 14 & 28 \\
\hline $1871-1880$ & 20 & 6 & 26 \\
\hline $1881-1890$ & 28 & 17 & 40 \\
\hline $1891-1900$ & 20 & 11 & 28 \\
\hline $1901-1914$ & 37 & 15 & 36 \\
\hline
\end{tabular}

Ce n'est donc pas un hasard si, à partir des années 1880, de nombreuses revues spécialisées comme la Revue internationale de l'enseignement ou la Revue pédagogique, à laquelle collaborent des pédagogues républicains comme Ferdinand Buisson, Ernest Lavisse ou Félix Pécaut, publient un nombre croissant de rapports et comptes rendus des missions ${ }^{14}$. Dimension souvent négligée par l'historiographie française, l'" international " constitue un cadre d'action et de pensée pour les réformateurs républicains ${ }^{15}$. Environ $35 \%$ de ces missions débouchent sur la publication d'une monographie relatant l'ensemble des observations et des analyses effectuées par le "chargé de mission ". Grâce à la richesse de cette production documentaire, l'étude des missions permet d'apporter une analyse originale de l'histoire de l'éducation en France.

Hormis un petit nombre de voyages personnels privés (11\% de l'échantillon), la mission est directement encadrée par le ministère de l'Instruction publique, que ce soit par la " commission des missions " (42\%) ou les différentes divisions de l'enseignement (47\%). Les missions peuvent être financées ou gratuites. Dans le premier cas, le ministère octroie au demandeur une subvention qui finance son voyage et ses frais de séjour. Lors d'une mission gratuite, le " chargé de mission " bénéficie des mêmes avantages diplomatiques que les membres de la première catégorie, mais sans aucune aide financière. L'intensification des missions pédagogiques à partir des années 1870, en termes absolus et par la diversité des destinations, s'accompagne de la systématisation d'une procédure

13 Les données présentées dans les tableaux et les graphiques de cet article ont été élaborés à partir de la base de données (cf. note 6).

14 Cf. Christophe Charle, La République des universitaires, 1870-1940, Paris, Seuil, 1994.

15 De nombreux aspects relatifs à ce champ réformateur ont été déjà bien étudiés, notamment le rôle de la franc-maçonnerie et des réseaux protestants. Cf. Jean-Paul Delahaye, "Les francs-maçons et la laïcisation de l'école. Mythe et réalités ", Histoire de l'éducation, n 109, 2006, p. 33-73, et André Encrevé, Michel Richard (dir.), Les Protestants dans les débuts de la Troisième République (1871-1885), Paris, Société d'histoire du protestantisme français, 1979. 
administrative apte à les encadrer. Les demandeurs envoient un dossier au ministre en exposant les motivations et les buts de leur projet, le choix de la destination et de l'objet d'étude dépendant de leurs intérêts et sensibilités. Le ministère ouvre alors un dossier et procède à une enquête minimale en vue d'obtenir des recteurs d'académie, des inspecteurs ou des directeurs d'établissement des informations sur la carrière et la " tenue " du requérant. La connaissance de la langue du pays de destination est une condition sine qua non de l'acceptation de la demande (seuls les inspecteurs peuvent bénéficier d'un traducteur qui les accompagne tout au long du voyage ${ }^{16}$ ).

À l'exception des connaissances linguistiques, il n'existe pas de critères de sélection fixés par des normes réglementaires. Les ancrages dans le monde institutionnel et ministériel, ainsi que l'originalité de la mission, constituent dès lors des atouts fort précieux, voire indispensables pour l'aboutissement d'un dossier. La carrière professionnelle et le réseau social des individus représentent des critères de sélection très importants, le capital social du demandeur influençant la décision du ministère. Ainsi, la présence de lettres de recommandation dans le dossier fournit un outil de légitimation supplémentaire lors de l'examen de la requête ${ }^{17}$. Faute de quoi, la méconnaissance ou l'anonymat scientifique du requérant condamne le dossier à une issue négative. Le cas de Jeanne Morin, publiciste qui s'intéresse aux questions de pédagogie, est exemplaire à cet égard. Désirant se rendre aux États-Unis en 1912 en vue d'étudier les jardins d'enfants dans les États de New York, Michigan et Columbia, elle voit sa demande refusée par le ministère. Sa mission n'apparaît pas apporter une contribution significative aux connaissances déjà acquises sur les jardins d'enfants en raison des nombreuses missions ayant étudié la même question en Amérique du Nord, mais également en Suisse, en Allemagne et en Italie dans les deux dernières décennies du XIX ${ }^{\mathrm{e}}$ siècle. De surcroît, l'ensemble des relations personnelles mobilisables dans son intérêt ne lui permet pas d'influencer la décision du ministère : "Mlle Morin n'a aucun dossier au ministère. Elle ne s'y est pas fait connaître "18. À ce capital social déficitaire et à la saturation des informations concernant son sujet d'étude, s'ajoutent le "vague " de l'objectif et le peu de

16 Cf. Gustave Salicis, Rapport à M. le Ministre de l'Instruction publique sur une mission relative à l'enseignement du travail manuel dans divers pays étrangers, Paris, 1887.

17 Dans de nombreux cas, des directeurs d'école, des inspecteurs, des recteurs d'académie, ainsi que des membres du Sénat, appuient avec véhémence la demande de mission d'un de leurs proches ou collaborateurs.

18 Archives Nationales (désormais AN), F/17/17280, dossier M ${ }^{\text {lle }}$ Morin Jeanne. 
consistance des moyens d'action qu'elle indique ${ }^{19}$. En revanche, si le profil correspond aux exigences ou aux attentes de la mission, le ministère octroie un financement et, par le biais du ministère des Affaires étrangères, fournit au demandeur des lettres de recommandation destinées au personnel consulaire du pays de destination. Cette démarche administrative permet de faciliter les recherches sur place et de fournir une pièce de légitimation aux yeux des futurs interlocuteurs du missionnaire. En effet, le statut officiel de " chargé de mission " est le seul qui permet d'ouvrir les portes des établissements scolaires, facilitant ainsi les visites et les recherches. Une fois la mission effectuée, le chargé de mission est tenu de fournir un rapport et, dans une minorité de cas, un compte rendu de l'utilisation exacte du financement ${ }^{20}$. Les rapports peuvent ensuite faire l'objet d'une publication sous forme de monographie ou être déposés dans leur version manuscrite aux archives du ministère.

\section{II - Une catégorisation des missions pédagogiques et des « chargés de mission »}

L'étude de la circulation internationale des idées requiert une attention particulière aux agents de l'échange intellectuel. Appliquée aux missions pédagogiques, une " sociologie des médiateurs ", jadis proposée par Michel Espagne et Michael Werner ${ }^{21}$, se révèle donc d'une grande importance pour examiner la diversité des profils professionnels des " chargés de mission " entre 1842 et 1914.

Tableau 2. Profession des "chargés de mission », 1842-1914.

\begin{tabular}{|l|c|}
\hline Profession & Pourcentage \\
\hline Inspecteurs de l'enseignement & 21 \\
\hline Professeurs de l'enseignement supérieur & 20 \\
\hline Professeurs de l'enseignement secondaire & 16 \\
\hline " Hommes de lettres ", publicistes & 12 \\
\hline Directeurs d'établissement scolaire (primaire et secondaire) & 5 \\
\hline Instituteurs & 4 \\
\hline Anciens élèves ENS, jeunes docteurs & 4 \\
\hline Fonctionnaires des ministères (autres que MIP) & 4 \\
\hline Autres & 14 \\
\hline Total & 100 \\
\hline
\end{tabular}

19 Id.

20 AN, F/17/17294, Mission Wolff.

21 Michel Espagne, Michael Werner, "La construction d'une référence culturelle allemande en France. Genèse et histoire (1750-1914) ", Annales ESC, n 4, 1987, p. 985. 
Les inspecteurs de l'enseignement sont les plus actifs, et une part analogue des missions est effectuée par les professeurs de l'enseignement supérieur. Il convient également de remarquer l'importance relative des professeurs de l'enseignement secondaire, des instituteurs des écoles primaires et des directeurs d'établissement (lycées, écoles primaires supérieures, etc.). Les " hommes de lettres ", essayistes et journalistes, sont finalement les seuls à ne pas relever directement du ministère de l'Instruction publique. Une analyse globale des missions montre de surcroît la grande diversité des objets d'étude.

Tableau 3. Répartition des objets d'étude, 1842-1914 ${ }^{22}$.

\begin{tabular}{|l|c|}
\hline Sujet d'étude & Pourcentage \\
\hline Instruction publique & 22 \\
\hline Enseignement primaire et populaire & 20 \\
\hline Enseignement secondaire & 12 \\
\hline Enseignement professionnel, technique, travail manuel & 12 \\
\hline Enseignement du dessin, écoles d'art & 6 \\
\hline Congrès et expositions nationales & 7 \\
\hline Salles d'asile, écoles maternelles, jardins d'enfants & 5 \\
\hline Enseignement des langues vivantes & 6 \\
\hline Éducation des femmes & 2 \\
\hline Autres & 8 \\
\hline Total & 100 \\
\hline
\end{tabular}

Les recherches à caractère général, touchant à l'instruction publique dans son ensemble, constituent l'objet d'étude le plus fréquent. Il est à supposer qu'étant donné le coût et la longueur des démarches administratives qu'elles impliquent, le " chargé de mission " en profite pour élargir au maximum le spectre de ses observations. À la préférence donnée à un cadre d'étude très large au moins jusque dans les années 1870, fait suite un éclatement progressif des sujets vers des problèmes plus spécifiques, liés à une branche scolaire (l'enseignement primaire et, dans une moindre mesure, l'enseignement secondaire et professionnel) ou à une discipline (dessin, langues vivantes, etc.). Dès lors, la répartition professionnelle des " chargés de mission " et la vaste gamme des sujets abordés permettent de subdiviser les 131 missions en trois catégories

22 Les catégories de classement ont été élaborées par l'auteur sur la base des objets d'étude des missions. Celles-ci se focalisent sur la visite des établissements scolaires, l'étude de leur organisation (contenu de l'enseignement, pratiques pédagogiques, etc.) et le recueil de documents statistiques et législatifs. 
reflétant, bien que schématiquement, l'hétérogénéité des profils sociaux, ainsi que les différentes fonctions du voyage.

\section{1 - Les missions pédagogiques : un réservoir d'exemples pour les réformes scolaires}

Le premier type de mission relève de la collecte d'informations en vue de la préparation d'une réforme scolaire. Dans la deuxième moitié du XIX ${ }^{e}$ siècle, les polémiques sur l'organisation de l'enseignement secondaire classique et moderne, la mise en place de l'obligation scolaire, des écoles maternelles, des premières filières de l'enseignement professionnel, ainsi que de la formation pédagogique des professeurs, s'appuient sur un faisceau d'expertises concernant les expériences étrangères. De ce fait, selon Jürgen Schriewer, les réformes scolaires de cette période se caractérisent par une "internationalisation sémantique " développée selon les exigences de chaque système national ${ }^{23}$. Ces missions représentent, de toute évidence, des instruments de légitimation qui accompagnent et appuient les remaniements du système éducatif. Elles réunissent la majorité relative des missions recensées et mobilisent trois profils professionnels : les professeurs universitaires, les inspecteurs et les " hommes de lettres".

Les professeurs des universités ou des Grandes écoles, en raison de leur capital culturel et de leur réseau de relations au sein du ministère, sont les plus actifs. Ils sont souvent rattachés aux facultés des lettres et bénéficient sporadiquement d'un traitement privilégié (par la délivrance d'un passeport diplomatique). À la demande du ministre Victor Duruy, Henry Montucci et Jacques Demongeot visitent en 1866 les établissements secondaires de plusieurs dizaines de villes anglaises. Leur séjour de deux mois est soigneusement préparé par la lecture des documents législatifs et des rapports publiés par les différentes commissions scolaires du gouvernement anglais. La mission débouche ensuite sur la publication d'une imposante monographie ${ }^{24}$. À cet égard, les rapports de Michel Bréal et de Georges Dumesnil sur le système scolaire allemand dans les

23 Hartmut Kaelbe, Jürgen Schriewer (dir.), Gesellschaften im Vergleich, Francfort, Peter Lang, 1998, p. 167.

24 Henri Montucci, Jacques Demongeot, De l'enseignement secondaire en Angleterre et en Écosse, Paris, Imprimerie impériale, 1868. 
années 1870 et $1880^{25}$, ainsi que les études de Célestin Hippeau sur l'état de l'instruction publique aux États-Unis et dans d'autres pays ${ }^{26}$, ne constituent que quelques-uns des exemples les plus connus. Le cas de Michel Bréal est particulièrement significatif : en identifiant dans le système scolaire français la source de la défaite militaire contre l'Allemagne en 1871, il utilise les observations formulées lors de ses voyages, ainsi que son expertise sur le sujet, afin d'intervenir dans le débat scolaire français ${ }^{27}$. La caractéristique principale de ce premier type de mission est sans doute l'exhaustivité des recherches, les études portant sur l'ensemble du système scolaire, ses différentes filières et son organisation générale. Le but est de fournir une vision globale du fonctionnement d'un système éducatif et d'identifier les éléments de comparaison les plus utiles à la France. Dotés du "droit au pouvoir symbolique " ${ }^{28}$, conféré par les missions et par leur statut professionnel, ces professeurs mènent alors une double carrière en tant que savants et réformateurs du système scolaire.

Par rapport aux professeurs de l'enseignement supérieur, les inspecteurs (21\%) effectuent des travaux d'expertise ponctuels, circonscrits à des thèmes et à des problèmes plus spécifiques. La mise en place de l'enseignement spécial par Victor Duruy est précédée, en 1863, d’une mission de Jean Magloire Baudouin, qui juge utile de consulter l'expérience de la Belgique, de la Suisse et de l'Allemagne dans ce domaine : "j'ai visité et habité ces contrées, j'en connais la langue, j'y ai conservé des relations, je suis peut-être dans des conditions exceptionnelles pour bien voir et tout voir et je rapporterai fidèlement [...] sans système préconçu, comme sans flatterie, tout ce que j'avais observé " ${ }^{29}$. Cette mission lui permet d'accéder deux ans plus tard au poste d'inspecteur général de l'Instruction publique pour l'enseignement primaire. Le rôle d'expert conféré par la mission est particulièrement important, car Baudouin prépare et publie en 1865 tous les programmes d'études pour la nouvelle filière de l'enseignement spécial. La mission peut ainsi conférer un " capital culturel international "

25 Michel Bréal, Excursions pédagogiques, $2^{2}$ éd., Paris, Hachette, 1884 ; Georges Dumesnil, La pédagogie dans l'Allemagne du Nord : les programmes, comment on les enseigne, comment on apprend à les enseigner, Paris, Delagrave, 1885.

26 Professeur de littérature à l'université de Caen entre 1847 et 1868. Une fois retraité, il entame dans les années 1870 une série d'études sur les systèmes éducatifs de l'Allemagne, de l'Angleterre, des États-Unis, de l'Italie, de l'Amérique du Sud, de la Russie et des pays scandinaves.

27 Michel Bréal, Quelques mots sur l'instruction publique en France, Paris, Hachette, 1873, p. 1.

28 Christophe Charle, Naissance des "intellectuels", 1880-1900, Paris, Éd. de Minuit, 1990, p. 8.

29 AN, F/17/2936, dossier Baudouin. 
convertible en titre professionnel ${ }^{30}$. Dans ce sens, à partir des années 1880 , un cercle d'inspecteurs relativement limité se manifeste en tant que véritable groupe d'experts. L'inspecteur de l'enseignement professionnel Guillaume Jost est mobilisé à plusieurs reprises. Entre 1878 et 1884, il se rend cinq fois en Allemagne afin de participer aux congrès des instituteurs et mener des visites dans les écoles professionnelles ${ }^{31}$. À la demande de Jules Ferry, Gustave Salicis voyage en Allemagne et dans les pays scandinaves durant toute l'année $1884^{32}$. Nommé inspecteur général à son retour, son expertise internationale fera de lui le promoteur de cet enseignement au niveau national à la fin des années 1880 . Le personnel féminin, à l'instar de Pauline Kergomard, inspectrice des écoles maternelles entre 1881 et 1917, est, en revanche, confiné au domaine de l'éducation de la petite enfance, étant chargé d'étudier les écoles maternelles, les jardins d'enfants et les salles d'asile ${ }^{33}$.

À ces missions d'expertise se superposent des voyages plus politiques, dont le but est de puiser des arguments pour mener une lutte à l'intérieur du cadre national. Il s'agit d'une action militante, ouvertement orientée vers le façonnement des réformes scolaires. Des publicistes et journalistes issus de plusieurs horizons politiques, tels que Jules Huret, Max Leclerc (directeur du Musée social) ou Edmond Dreyfus-Brisac (rédacteur en chef de la Revue internationale de l'enseignement), tirent argument du résultat de ces missions pour soutenir leur vision des réformes. Les institutions ou sociétés d'études auxquelles ils appartiennent servent ensuite de caisse de résonance, à travers la publication d'articles et de brochures, ce qui contribue fortement à introduire une dimension internationale dans les polémiques scolaires françaises ${ }^{34}$.

30 Yves Dezalay, "Les courtiers de l'international. Héritiers cosmopolites, mercenaires de l'impérialisme et missionnaires de l'universel ", Actes de la recherche en sciences sociales, n 151-152, 2004, p. 5-35.

31 AN, F/17/10799, dossier Jost.

32 AN, F/17/10799, dossier Salicis.

33 Pauline Kergomard, Exposition universelle de 1889. Monographies pédagogiques. Les écoles maternelles (anciennes salles d'asile), Paris, Imprimerie nationale, 1889.

34 Edmond Dreyfus-Brisac, L'éducation nouvelle. Études de pédagogie comparée, Paris, Masson, 1888, p. 231. En effet, de nombreuses revues (Revue internationale de l'enseignement, Revue pédagogique, Revue des deux mondes, etc.) publient régulièrement des articles portant sur les systèmes éducatifs étrangers. 


\section{2 - Missions pédagogiques et " savoir-faire " professionnel}

Le deuxième type de mission se situe au carrefour entre une mission proprement dite et le voyage d'étude personnel. Englobant les instituteurs, les directeurs d'établissement et les professeurs de l'enseignement secondaire, ces missions représentent plutôt une sorte de récompense pour l'exemplarité de leur carrière, le travail accompli au sein de leur propre école ou leur fidélité à l'égard de l'administration. La demande formulée en 1880 par le directeur de l'école communale de Saint-Martin à Périgueux est accueillie favorablement par le ministre, qui souligne son zèle et son dévouement dans la direction de son établissement :

" Je suis heureux de vous témoigner toute ma satisfaction et si vous étiez disposé à aller aux vacances prochaines étudier l'organisation pédagogique de la Suisse, je vous en faciliterais les moyens en vous accordant une indemnité de voyage et de séjour. Je suis convaincu qu'un maître tel que vous, ne pourrait pas manquer de mettre à profit les observations qu'il recueillerait dans une mission de cette nature ${ }^{35}$.

Ce deuxième type de mission se distingue par son caractère intime, le voyage visant plutôt l'amélioration du savoir-faire professionnel d'un personnel enseignant sélectionné au préalable. En effet, les maîtres et les professeurs, pendant leurs vacances, partent à l'étranger en vue d'étudier telle ou telle école, ou tel ou tel procédé pédagogique, mais surtout pour se perfectionner dans l'exercice de leur métier. De retour d'un voyage d'étude aux écoles de travail manuel de Naas, en Suède, l'instituteur parisien Théodore Petit remercie le ministère d'avoir pu profiter de cette possibilité d'acquérir de nouvelles connaissances, qu'il s'efforcera "d'appliquer [...] suivant les désirs de l'Administration " ${ }^{36}$. Intéressé par les méthodes en vigueur dans les écoles techniques suisses, R. Rouffié, professeur à l'école primaire supérieure de Toulon, prie le ministre de lui accorder une mission afin de pouvoir " en retirer un profit solide, au point de vue de l'expérience professionnelle ${ }^{37}$. La rédaction d'un rapport ou d'un compte rendu ne constitue pas la priorité d'un tel type de voyage, qui demeure relativement autonome par rapport à l'agenda des réformes scolaires. En effet, le capital culturel de ces acteurs ne semble pas être suffisant pour que leurs éventuelles propositions puissent s'affirmer sur le plan national.

35 AN, F/17/9393/A, dossier Deschamps.

36 AN, F/17/10799, rapports missions d'instituteurs.

37 AN, F/17/3004/A, mission Rouffié. 
La restitution de l'expérience en terre étrangère se limite, le cas échéant, à la rédaction de quelques articles pour des revues spécialisées, notamment la Revue pédagogique. Dans certains cas, le comité de rédaction de la revue refuse la publication d'un compte rendu en raison de la "médiocrité intellectuelle " du chargé de mission ${ }^{38}$.

\section{3 - Missions pédagogiques et propagande culturelle}

Le dernier type de mission concerne la "diplomatie scolaire " et les missions de représentation, voire de propagande, du système éducatif français lors des expositions universelles ou des congrès d'instituteurs et professeurs à l'étranger. Peu nombreuses (moins de $10 \%$ de l'échantillon), ces missions sont pourtant significatives, en raison de la multiplication de ce genre d'événement dans le dernier tiers du XIX ${ }^{\mathrm{e}}$ siècle. Lieux d'exhibition de la puissance nationale, les expositions universelles présentent dès 1862 des sections permanentes consacrées à l'enseignement et aux méthodes pédagogiques. Plus précisément, les États y exposent l'organisation de leur propre système scolaire, le contenu des enseignements, les travaux des élèves, les plans architecturaux d'écoles modèles, etc. Selon Eckhardt Fuchs, les sections scolaires ont donc pour but premier de vanter les mérites des systèmes éducatifs nationaux ${ }^{39}$. Le ministère de l'Instruction publique saisit très vite l'importance de ces événements dans une optique d'expansion de l'influence culturelle française à travers le monde. Ce sont les chefs de division du ministère, les inspecteurs généraux ou des membres du Conseil supérieur de l'instruction publique qui sont chargés d'organiser la section française et, le cas échéant, de visiter les écoles du pays organisateur. Ferdinand Buisson se construit, ce faisant, un véritable prestige national et international ${ }^{40}$. Bien qu'émargeant au ministère de l'Instruction publique, il occupait jusque-là une position subordonnée et relativement contestataire. Son engagement au cours des expositions universelles fait partie d'une stratégie consciente de promotion d'un système scolaire gratuit, obligatoire et laïque, qui lui permet, de surcroît, d'accéder aux plus hautes

38 AN, F/17/9393/B, mission Hasselot.

39 Eckhardt Fuchs, "Educational Sciences, Morality and Politics : International Educational Congresses in the Early Twentieth Century ", Paedagogica Historica, vol. 40, n 5-6, 2004, p. 759.

40 Ses rapports sur les sections scolaires des expositions universelles de 1873 à Vienne et de 1876 à Philadelphie connaissent une diffusion européenne. 
sphères de l'administration française ${ }^{41}$ : il sera directeur de l'enseignement primaire entre 1879 et 1896 . Son frère Benjamin, ancien professeur de langue française à Londres et directeur de l'enseignement primaire à Tunis entre 1890 et 1905, est chargé d'organiser les expositions de La Nouvelle-Orléans (1884) et de Melbourne (1888) et participe aux expositions de Chicago en 1893 et de Saint-Louis en 1904. Ces dernières sollicitent également d'autres personnalités bien connues, comme Jules Steeg, proche de Ferdinand Buisson et directeur du Musée pédagogique, ainsi que son remplaçant Athanase Édouard Gilles, nommé inspecteur général de l'enseignement primaire après le décès de Steeg en 1898. Moments de comparaison et d'autoréflexivité par excellence, les sections scolaires des expositions universelles constituent un moment privilégié pour les contacts entre les spécialistes de la pédagogie à l'échelle mondiale. D'autre part, elles représentent un instrument de légitimation des politiques scolaires républicaines, qui trouvent ainsi une assise internationale les protégeant de leurs détracteurs.

\section{III - Les missions pédagogiques et la construction de modèles scolaires}

L'étude des missions pédagogiques se confronte à un problème complexe, celui de la fonction et de l'impact du voyage sur les politiques scolaires nationales. Quel que soit le type de mission, les "chargés de mission " recherchent dans les pays étrangers des exemples, des méthodes et des idées jugés plus novateurs ou efficaces par rapport au système domestique du point de vue pédagogique ou administratif ${ }^{42}$. Afin de savoir comment se construisent des modèles scolaires de référence, il est donc important d'examiner où se dirigent prioritairement les chargés de mission, ainsi que les critères de sélection des destinations.

41 Klaus Dittrich, "La modernisation du système éducatif à travers les Expositions universelles, 1873-1904 ", à paraître dans Japon pluriel 8, Actes du huitième colloque de la Société française des études japonaises, Arles, Éd. Philippe Picquier.

42 Comme le fait remarquer Benjamin Buisson, tout ne se prête pas à l'imitation : " notre intention n'est pas de proposer l'imitation servile ni l'importation de toutes pièces d'aucune des coutumes de l'étranger, mais de provoquer, par la comparaison, des réflexions qui ne peuvent manquer d'être fructueuses ", L'enseignement primaire aux Congrès d'éducation et à l'Exposition scolaire de Chicago, Paris, Hachette, 1896, p. 186. 
Tableau 4. Pays de destination des missions, 1842-1914.

\begin{tabular}{|l|c|}
\hline Pays & Pourcentage \\
\hline Allemagne (ensemble) & 21 \\
\hline États-Unis & 15 \\
\hline Suisse & 14 \\
\hline Angleterre, Écosse, Irlande & 11 \\
\hline $\begin{array}{l}\text { Pays Scandinaves } \\
\text { (Norvège, Suède, Finlande, Danemark) }\end{array}$ & 9 \\
\hline Autriche-Hongrie & 6 \\
\hline Italie & 6 \\
\hline Grèce & 3 \\
\hline Autres & 15 \\
\hline Total & 100 \\
\hline
\end{tabular}

Avec $21 \%$ des missions, l'Allemagne se situe en tête, suivie des États-Unis, de la Suisse et, nettement distancées, de l'Angleterre et des pays scandinaves. À l'exception des États-Unis, les missions s'inscrivent dans un cadre géographique essentiellement européen. Sur un total de vingt-sept pays, en effet, seuls le Brésil, le Japon, l'Égypte, l'Australie, le Canada, la Turquie et la Syrie font l'objet d'une mission ou plus. L'importance de l'Allemagne n'est guère surprenante car, dans la deuxième moitié du XIX siècle, son modèle scolaire s'affirme comme un véritable point de repère aux yeux de bon nombre de réformateurs français et européens ${ }^{43}$. Cet exemple est sollicité comme une double référence : d'une part, en tant que modèle général de réussite scientifique, grâce au grand prestige des universités allemandes, et, d'autre part, comme modèle spécifique en matière d'enseignement secondaire et professionnel. En France, l'issue de la guerre de 1870-1871 représente un point de rupture symbolique important. Après Sedan, le thème de l'instruction déferle dans les gazettes françaises, et l'instituteur allemand est identifié comme le véritable vainqueur de la guerre. Cet événement façonne ce que Claude Digeon désignera plus tard comme la " question allemande ", c'est-à-dire un complexe psychologique mêlant des sentiments de rivalité et d'admiration envers le voisin d'outre-Rhin ${ }^{44}$. L'état de l'instruction publique à tous ses degrés, avec la mauvaise organisation de l'armée et l'incompétence gouvernementale, entre de droit parmi les facteurs

43 Monique Mombert, L'enseignement de l'allemand en France. Entre "modèle allemand " et "langue de l'ennemi ", Strasbourg, Presses universitaires de Strasbourg, 2001.

44 Claude Digeon, La crise allemande de la pensée française, Paris, Presses universitaires de France, 1959, p. 1. 
responsables de la "dégénérescence "française ${ }^{45}$. À cet égard, l'inspecteur Alfred Picard, retraçant a posteriori les effets et le pouvoir structurant de cet événement pour l'histoire de l'enseignement en France, souligne comment " on répéta comme un axiome que, si nous avions été battus par des troupes supérieures en nombre et mieux commandées, nous le devions surtout à l'infériorité de nos écoles. Ce sentiment général, qu'elle qu'en fût la valeur, exerça une action puissante sur le développement de l'instruction en France dans les années qui suivirent $"^{46}$.

Les références permanentes à l'Allemagne ne doivent pourtant pas cacher le rôle des autres modèles. Les États-Unis, puissance mondiale émergente, recueillent notamment un nombre de visites fort important, grâce également aux quatre expositions universelles qui se déroulent sur leur territoire dans les trois dernières décennies du siècle $(1876,1884$, 1893 et 1904). Petit pays au grand prestige pédagogique, la Suisse fait figure d'excellence dans ce tableau. Jouissant depuis le début du XIX ${ }^{\mathrm{e}}$ siècle d'un taux d'alphabétisation très élevé, elle représente l'une des destinations privilégiées des voyageurs français : la diversité des méthodes et des théories pédagogiques en vigueur dans les différents cantons font d'elle un véritable laboratoire d'expériences ${ }^{47}$. L'étendue et l'hétérogénéité du réseau scolaire primaire et les grandes figures de la pédagogie suisse (Pestalozzi ou le Père Girard) captivent les observateurs français. Le professeur d'hygiène de la faculté de médecine de Montpellier Jean-Baptiste Fonssagrives livre ainsi un récit positif de sa visite dans la Confédération helvétique en 1871 : "Je rapporte de ce voyage l'impression générale que nous avons beaucoup à emprunter à la Suisse pédagogique. Elle constitue [...] une sorte d'exposition scolaire permanente où toute l'Europe se donne rendez-vous et que nous ne saurions interroger trop attentivement " ${ }^{48}$. Cela dit, une analyse diachronique des pays de destination permet d'émettre quelques hypothèses sur le poids de chaque modèle, en évitant le piège de la surreprésentation de l'Allemagne dans les configurations discursives de l'époque.

\footnotetext{
45 Ibid., p. 364.

46 Alfred Picard, Exposition universelle internationale de 1889 à Paris. Rapport général, Paris, Imprimerie nationale, 1890 , p. 357.

47 AN, F/17/2988, mission Joseph Marmier.

48 AN, F/17/12340, échanges avec l'étranger, Suisse.
} 


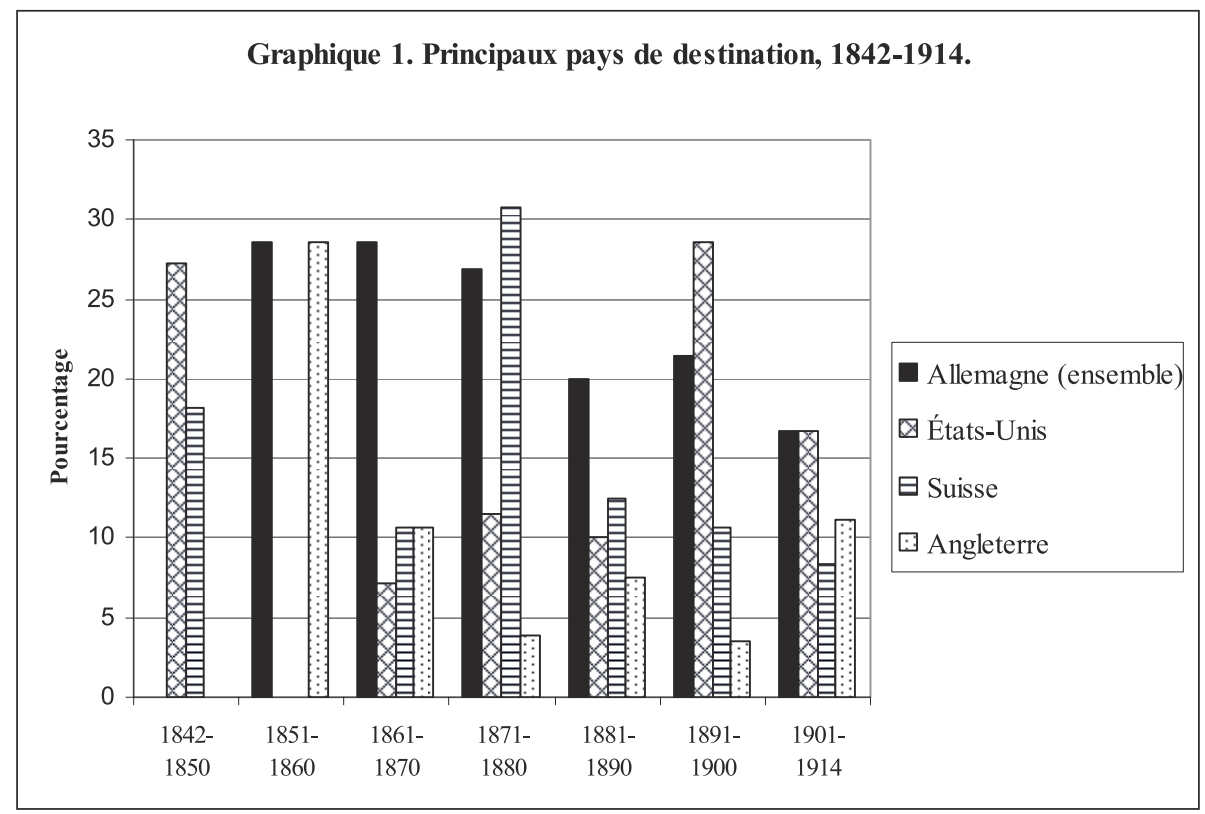

Deux éléments méritent d'être soulignés : l'intérêt pour l'Allemagne bien avant la guerre de 1870-1871 et l'importance relative d'autres modèles scolaires. Tout en conservant toujours la première place, la part de l'Allemagne diminue progressivement au profit de celle des États-Unis, qui prédominent d'ailleurs dans les années 1890. Le poids de la Suisse demeure toujours significatif, tandis que l'Angleterre, hormis pendant la période 1850-1870, occupe une place très modeste. Pour expliquer ce mouvement, il est indispensable de se pencher sur les raisons qui amènent les "chargés de mission " à sélectionner un pays plutôt qu'un autre. En se focalisant sur les quatre destinations principales et en les croisant avec les sujets de recherche consacrés à une filière scolaire précise, nous obtenons un résultat qui éclaire le tableau précédent. 


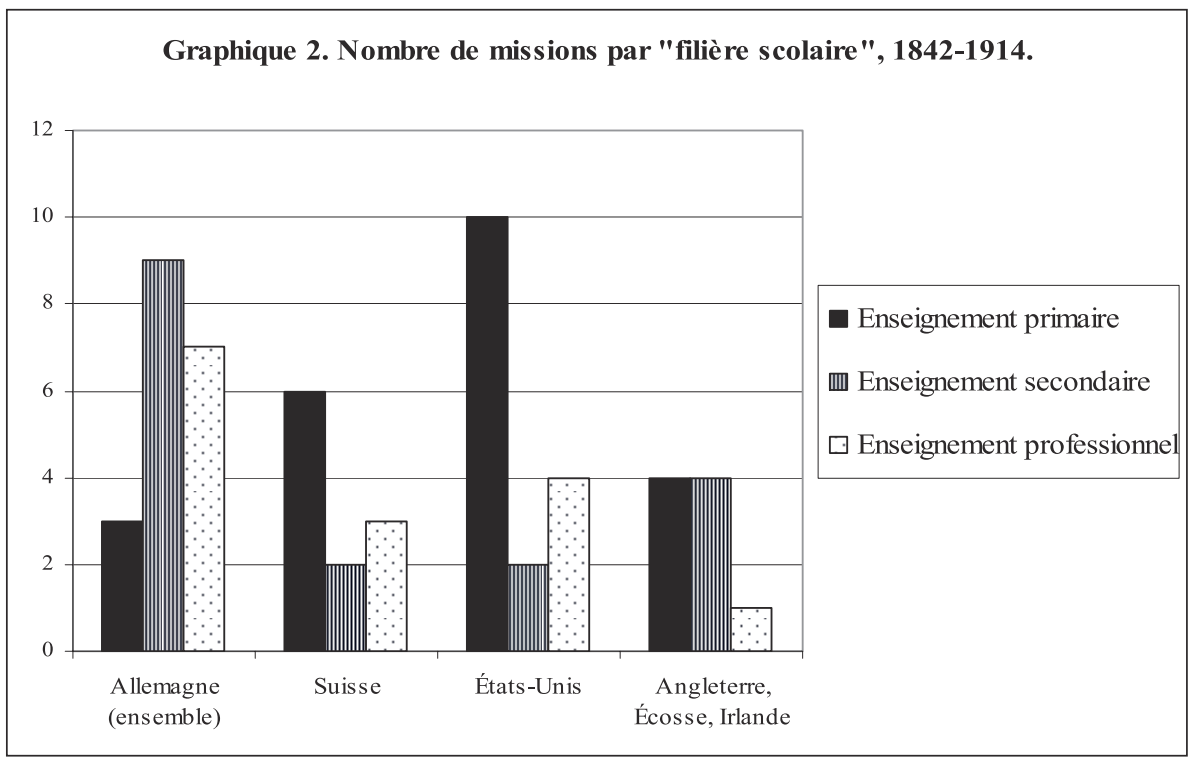

L'opposition principale se joue entre l'Allemagne et les États-Unis et se structure autour des deux niveaux qui se trouvent à la base des systèmes scolaires modernes, à savoir l'enseignement primaire et l'enseignement secondaire. La sélection du pays de destination est alors soumise à d'autres variables, qui ne relèvent pas nécessairement de la pure pédagogie. Premièrement, une variable macro-économique. Dans le contexte de la deuxième révolution industrielle, le poids économique international croissant de l'Allemagne (et, dans une moindre mesure, celui des États-Unis) peut contribuer à expliquer son attrait et sa suprématie en matière d'enseignement professionnel et technique. En tant que rivale impériale directe, les " chargés de missions " y cherchent des exemples en mesure d'améliorer la formation d'acteurs économiques compétents. Deuxièmement, une variable politique. Les " chargés de mission " se soucient des principes politiques régissant un modèle de système éducatif conforme à l'idéal démocratique et républicain ${ }^{49}$. Le désintérêt pour l'Angleterre s'inscrit, certes, dans le contexte du relatif déclin économique de ce pays dans la deuxième moitié du XIX ${ }^{\mathrm{e}}$ siècle, mais s'explique aussi par son organisation scolaire laissant une grande place à l'initiative privée et religieuse. Il s'agit d'un modèle d'éducation élitiste, qui n'est pas conforme à l'idéologie républicaine.

49 Pierre Caspard, "Les miroirs réfléchissent-ils ? Esquisse d'une étude comparée de la gratuité, de l'obligation et de la laïcité scolaires, en France et en Suisse ", in Rita Hofstetter et al. (dir.), Une école pour la démocratie. Naissance et développement de l'école primaire publique en Suisse au XIX ${ }^{e}$ siècle, Berne, Peter Lang, 1999, p. 344. 
En revanche, le self-government et l'idéal politique incarnés par le free school system peuvent expliquer, du moins en partie, l'intérêt particulier accordé à l'instruction primaire états-unienne, qui se traduit par leur primauté dans les missions consacrées à ce niveau d'enseignement. En effet, l'obligation scolaire pour le peuple, en vue de construire sa citoyenneté politique, implique des soubassements politiques fort importants. En 1900, Charles-Victor Langlois souligne les liens étroits entre systèmes scolaire et politique en montrant comment des pays ayant une structure politique " aristocratique ", comme l'Angleterre et l'Allemagne, s'opposent à des " démocraties " comme la Suisse et les ÉtatsUnis, ou à des régimes politiques " hybrides " tel celui de la France ${ }^{50}$. L'accès au " savoir " et à une citoyenneté politique rêvée est un thème central de l'idéologie républicaine. Au-delà de l'Atlantique, "l'instruction est la condition essentielle de l'existence d'un peuple libre "51, et l'imitation de l'exemple américain, indique Célestin Hippeau, pourrait stabiliser politiquement le nouveau gouvernement républicain ${ }^{52}$. D’après lui, le système scolaire américain s'est constitué à partir du principe qu'Alexis de Tocqueville a identifié comme celui de la " souveraineté du peuple ", qui surplombe tout le système politique. Ferdinand Buisson, après l'exposition de Philadelphie de 1876 et la visite des établissements scolaires de douze villes américaines, souligne l'importance des soubassements politiques dans la mise en place et les progrès de l'instruction publique américaine ${ }^{53}$. Les particularités de ce système scolaire, notamment la décentralisation de la gestion et de l'administration des écoles, ainsi que, du point de vue strictement pédagogique, la " coéducation " des sexes (à savoir l'enseignement commun aux filles et aux garçons à tous les échelons de l'instruction), ne seraient donc possibles que dans une société pénétrée par l'esprit démocratique ${ }^{54}$.

En revanche, l'enseignement secondaire, dévolu dans l'idéologie républicaine à la construction d'une élite sociale, ne répond que très partiellement à l'imaginaire démocratique qu'on trouve au fondement de l'enseignement

50 Charles-Victor Langlois, Rapports du jury international - Étranger, Paris, Imprimerie nationale, 1900, p. 101-114.

51 Célestin Hippeau, L'instruction publique en Allemagne, Paris, Didier, 1873, p. 17.

52 Célestin Hippeau, L'instruction publique aux États-Unis. Écoles publiques, collèges, universités, écoles spéciales, rapport adressé au ministre de l'instruction publique, $2^{\mathrm{e}}$ éd, Paris, Didier, 1878, p. 2.

53 Ferdinand Buisson, Rapport sur l'instruction primaire à l'Exposition universelle de Philadelphie en 1873, présenté à M. le ministre de l'Instruction publique au nom de la commission envoyée par le ministère à Philadelphie, Paris, Imprimerie nationale, 1878, p. 2-20.

54 James Albisetti, "European Perceptions of American Coeducation, 1865-1914: Ethnicity, religion and culture ", Paedagogica Historica, vol. 37, n 1, 2001, p. 123-138. 
primaire. Dès lors, un système " aristocratique " comme celui de l'Allemagne ${ }^{55}$ se prête particulièrement bien à la comparaison et, par conséquent, à l'étude des emprunts possibles. Pendant la " crise de l'enseignement secondaire " qui parcourt toute la deuxième moitié du XIX ${ }^{\mathrm{e}}$ siècle, l'Allemagne demeure sans doute la référence principale. L'exemple de la Realschule est sollicité à maintes reprises : l'enseignement des "réalités " (langues vivantes et sciences) et la spécialisation " utilitariste " précoce des études secondaires contribuent à établir un équilibre entre les études " classiques " et " modernes ", c'est-à-dire entre la reproduction de l'ordre social et les nouvelles exigences économiques. Fondée par Jean-Jules Hecker en 1747, la Realschule se veut donc, comme le souligne le Dictionnaire de pédagogie, une école apte à " satisfaire aux besoins de la bourgeoisie devenue plus forte, et présente une éducation appropriée aux conditions d'existence des classes moyennes auxquelles la Volksschule offrait trop peu, tandis que l'école latine leur offrait trop et que son enseignement n'avait pas d'utilité pratique " ${ }^{56}$. Si la Realschule et l'enseignement secondaire allemand cristallisent particulièrement l'attention des réformateurs français, c'est parce que la querelle entre " classiques " et "modernes ", et les polémiques sur les contenus et les débouchés de l'enseignement secondaire, sont consubstantielles aux enjeux de la fabrication de l'élite sociale. La réception du concept de Realschule en France contribue donc à façonner une structure scolaire qui portera le nom d'enseignement spécial (1865). C'est le ministre Léon Bourgeois qui transforme en 1891 l'enseignement spécial en enseignement " moderne ", avec plein accès au baccalauréat et aux universités (à l'exclusion des facultés des sciences et de médecine). La réforme de 1902 débouche finalement sur l'égalité de sanction entre enseignements classique et moderne, situation à laquelle l'Allemagne était parvenue une dizaine d'années auparavant. Conjointement à la mise en place de ces filières, de nombreuses missions visitent à plusieurs reprises les établissements secondaires allemands. La différenciation marquée de l'offre scolaire au niveau secondaire est louée à maintes reprises par les " chargés de mission ". Dans ce sens, selon Edmond Dreyfus-Brisac, l'Allemagne (et notamment la Prusse) est " peut-être de tous les pays celui où la séparation - tant du point de vue administratif qu'au point de vue pédagogique - entre

55 Guillaume Vaubert, "L'empereur Guillaume II et ses vues sur la réforme de l'enseignement secondaire ", Revue des Deux Mondes, 1891, t. 103, vol. 1, p. 208.

56 Ferdinand Buisson (dir.), Nouveau dictionnaire de pédagogie et d'instruction primaire, Paris, Hachette, 1911, entrée "Allemagne ". 
les trois ordres d'enseignement : primaire, secondaire, supérieur, est la plus profonde et la mieux tranchée ${ }^{57}$. L'enseignement secondaire allemand offre finalement un modèle qui conjugue diversification de l'offre scolaire, sélection sociale et adaptation aux nouvelles exigences économiques.

Les missions pédagogiques représentent l'un des vecteurs autour desquels se construit l'internationalisation du discours pédagogique de la deuxième moitié du XIX ${ }^{e}$ siècle. Plus qu'à la mise en place d'un "internationalisme gouvernemental " 58 , l'intensification des missions à partir des années 1870 constitue la réponse institutionnelle à une demande sociale formulée par une nébuleuse d'acteurs aux profils multiples, variant du professeur universitaire à l'instituteur de province, et disposant d'une marge de manœuvre quant au choix de la destination et de l'objet d'étude. Sur le plan national, les expériences étrangères fonctionnent essentiellement comme des réservoirs d'expériences, qui sont modulées en fonction des avantages comparatifs des différents pays : la hiérarchie des pays de destination révèle des modèles scolaires construisant un système de référence exploité différemment en fonction d'enjeux politiques, qui se superposent souvent aux questions pédagogiques. Quant aux effets des missions sur les politiques scolaires françaises, ils se mesurent moins par des phénomènes de transferts que par " la mise en place de cadres de pensées, de réseaux d'acteurs, de schèmes d'organisation ou de lieux de production du savoir " 59 : les missions offrent un faisceau d'observations et de renseignements savants sur de nombreux problèmes de l'instruction publique. Du point de vue des " chargés de mission ", le savoir sur l'étranger, ainsi que les modalités de sa production - la mission -, fournissent un " capital international " mobilisable lors des polémiques scolaires, d'une part, et dans le cadre de stratégies professionnelles ascendantes, d'autre part. L'exemple des inspecteurs de l'enseignement est à cet égard éclairant : un bagage de connaissances, d'expériences et de contacts personnels au niveau international constitue un atout précieux pour l'obtention d'un poste. L'intégration d'un horizon de références dépassant

57 Edmond Dreyfus-Brisac, "Les principes régulateurs de l'enseignement en Prusse ", Revue internationale de l'enseignement, 1884, p. 386.

58 Madeleine Herren, Hintertüren zur Macht. Internationalismus und modernisierungsorientierte Außenpolitik in Belgien, der Schweiz und den USA, 1865-1914, Münich, R. Oldenbourg, 2000.

59 Pierre-Yves Saunier, "Circulations, connexions et espaces transnationaux ", Genèses, 2007, n 57, p. 115. 
le cadre national opère donc comme une forme de légitimation savante. Enfin, la conjugaison de l'expertise scolaire avec le souci comparatif, exercice qui n'est d'ailleurs pas circonscrit à la seule France, montre à quel point la dimension internationale constitue un élément central dans la construction du système scolaire moderne, voire de l'État-Nation.

\section{Damiano MATASCI}

Université de Genève, EHESS damiano.matasci@unige.ch 